Licença CC BY: Artigo distribuído sob os termos Creative Commons, permite uso e distribuição irrestrita em qualquer meio desde que 0 autor credite a fonte original.

\section{REPRESENTATIVIDADE FEMININA NA ÁREA ACADÊMICA DE TURISMO: UMA ANÁLISE DOS PROGRAMAS DE PÓS- GRADUAÇÃO FILIADOS À ANPTUR}

\author{
FEMALE REPRESENTATIVENESS IN THE ACADEMIC TOURISM \\ AREA: AN ANALYSIS OF GRADUATE PROGRAMS AFFILIATED TO \\ ANPTUR
}
REPRESENTATIVIDAD FEMENINA EN EL ÁREA ACADÉMICA DE TURISMO: UN ANÁLISIS DE LOS PROGRAMAS DE POSGRADO AFILIADOS A ANPTUR

\author{
MARIANA DE FREITAS COELHO 1 \\ VERÔNICA FEDER MAYER 2 \\ MARIANA BUENO DE ANDRADE-MATOS 3 \\ DANIELA FANTONI ALVARES 4
}

${ }^{4}$ UNIVERSIDADE DOS AÇORES, PONTA DELGADA - ILHA DE SÃO MIGUEL, PORTUGAL

DATA DE SUBMISSÃO: 28/12/2020 - DATA DE ACEITE: 19/06/2021

\begin{abstract}
RESUMO: Existem fatores que interferem na atuação docente relacionados a questões ligadas aos sexos, mas faltam estudos no contexto de Programas de Pós-Graduação em Turismo (PPGTurs). O objetivo deste artigo é compreender como a inserção feminina de docentes de turismo acontece no âmbito da Associação Nacional de Pesquisa e Pós-Graduação em Turismo (Anptur). A pesquisa é quantitativa, baseada em dados secundários e análises estatísticas utilizando o software SPSS. Os resultados apontam equilíbrio entre o número de docentes dos dois sexos atuando em programas de pós-graduação em turismo. Os cargos de coordenação e vice coordenação dos PPGTurs têm maioria masculina (60\%), assim como em cargos de diretoria da Anptur (62\%). Há diferenças na média de citação de mulheres, que é inferior à citação de homens em todos os índices existentes na plataforma Google Scholar. Sobre a autoria de trabalhos publicados em anais da Anptur entre 2005 e 2019, tem-se que há maioria de autoras do sexo feminino (60\%). Sobre a premiação, há um número equilibrado de prêmios concedidos aos dois sexos. 0 estudo contribui ao apresentar um recorte sobre a atuação docente em programas de pós-graduação em turismo, colaborando para o entendimento da representatividade e influência feminina na área.
\end{abstract}

Mariana: Professora do Departamento em Administração e Contabilidade da Universidade Federal de Viçosa, Viçosa, Minas Gerais, Brasil. Doutora em Administração pela Universidade Federal de Minas Gerais, Belo Horizonte, Minas Gerais, Brasil. E-mail: marifcoelho@gmail.com.http://orcid.org/0000-0002-7081-1429.

Verônica: Professora associada da Faculdade de Turismo e Hotelaria da Universidade Federal Fluminense (UFF), Niterói, RJ, Brasil. Doutora em Administração pela Universidade Federal do Rio de Janeiro, Rio de Janeiro, RJ Brasil. E-mail: veronicamayer@id.uff.br. https://orcid.org/0000-0002-7543-5215.

Mariana: Professora Doutora no bacharelado em Lazer e Turismo e no Programa de Pós-Graduação em Turismo (PPGTUR/USP) na Escola de Artes, Ciências e Humanidades da Universidade de São Paulo (EACH/USP), São Paulo, São Paulo, Brasil. Doutora em Administracão pela Universidade Federal de Pernambuco, Brasil. E-mail: buenomariana@usp.br. https://orcid.org/0000-0002-8723-3258.

Daniela: Professora Auxiliar em Turismo na Universidade dos Açores, Ponta Delgada - Ilha de São Miguel, Portugal. Doutora em Planejamento, Universidade do Minho, Guimarães, Portugal. E-mail: daniela.f.alvares@gmail. com. Orcid:https://orcid.org/0000-0002-2433-0448. 
PALAVRAS-CHAVE: representatividade feminina; docentes; igualdade de gênero; programas de pósgraduação em turismo.

\begin{abstract}
Some factors related to gender affect teaching performance, but studies in the context of Postgraduate Programs in Tourism (PPGTurs) are still lacking. This article aims to understand the representativeness of female teachers of tourism within the scope of the National Association for Research and Postgraduate Studies in Tourism (Anptur - Brazil). This research is quantitative, based on secondary data and statistical analysis using the software SPSS. The results indicate a balance between the number of professors of both sexes working in postgraduate programs in tourism. The coordination and vice-coordination positions of the PPGTurs are mostly occupied by males (60\%), as are the majority of management positions at Anptur (62\%). There are differences in the average number of women, which is lower than that of men in all indices on the Google Scholar platform. Concerning the authorship of works published in Anptur's annals between 2005 and 2019, there is a majority of female authors (60\%). Regarding awards, the number of awards received by men and women was roughly the same. This study contributes by presenting an excerpt on teaching practice in postgraduate programs in tourism, contributing to an understanding of representativeness and female influence in the area.
\end{abstract}

KEYWORDS: female representativeness; gender equality; faculty member; graduate programs in tourism.

RESUMEN: Existen factores que interfieren en el desempeño docente relacionados con sus sexos, pero faltan estudios en el contexto de los Programas de Posgrado en Turismo (PPGTurs). El propósito de este artículo es comprender cómo se da la inserción femenina de profesoras de turismo en el ámbito de la Asociación Nacional de Investigación y Posgrado en Turismo (Anptur - Brasil). La investigación es cuantitativa, basada en datos secundarios y análisis estadístico utilizando el software SPSS. Los resultados indican un equilibrio entre el número de profesores de ambos sexos que trabajan en programas de posgrado en turismo. Los puestos de coordinación y vicecoordinación de los PPGTurs tienen mayoría masculina (60\%), así como en los puestos directivos de Anptur (62\%). Existen diferencias en el número medio de citas de mujeres, que es inferior al de los hombres en todos los índices de la plataforma Google Scholar. Sobre la autoría de las obras publicadas en los anales de Anptur entre 2005 y 2019, hay una mayoría de autoras (60\%). En cuanto al premio, existe un número equilibrado de premios otorgados a ambos sexos. El estudio contribuye presentando un extracto sobre el desempeño docente en programas de posgrado en turismo, contribuyendo a la comprensión de la representatividad e influencia femenina en la zona.

PALABRAS CLAVE: representatividad femenina; igualdad de género; miembro de facultad; programas de posgrado en turismo.

\title{
INTRODUÇÃO
}

Não recentemente, a luta e a necessidade de igualdade de direitos e oportunidades entre os sexos passaram a ser discutidas social e academicamente. Ao longo da história da humanidade, a mulher conquistou o direito ao acesso à vida política, à educação, ao divórcio e ao mercado de trabalho, por exemplo. No entanto, dificuldades para uma efetiva igualdade entre os sexos existem, tais como a divisão de tarefas domésticas, a parceria na educação de filhas e filhos, preconceitos e assédio.

Em âmbito mundial, o debate tem crescido, apesar de ainda haver inúmeros desafios envolvidos nas questões de gênero, que permeiam questões culturais, sociais, religiosas e políticas. A relevância dessa discussão pode ser percebida inclusive na Agenda 2030, desenvolvida pela Organização das Nações Unidas, a qual definiu 17 Objetivos de Desenvolvimento Sustentável 
(ODS), sendo que um destes é exatamente a "Igualdade de Gênero". Neste sentido, há, em diversas regiões do mundo, ações em andamento e um esforço para a consecução deste objetivo. Lima (2013), tratando especificamente da participação das mulheres na ciência na área de computação, afirma que há discriminações e segregações sutis existentes que exigem das mulheres um esforço a mais para conseguirem atingir o mesmo sucesso de um homem. Apesar das dificuldades não serem necessariamente as mesmas para todas as áreas, estudos apontam que desafios também existem nas áreas profissional e acadêmica de turismo (Nunkoo et al., 2020). No contexto dos cursos de turismo, Minasi e Censon (2020) indicam que 69,1\% dos discentes em cursos de graduação são do sexo feminino, ao passo que $30,9 \%$ é do sexo masculino. A maioria feminina também é indicada pelas autoras em relação aos discentes de programas de mestrado e doutorado acadêmicos na referida área (Minasi \& Censon, 2020).

Apesar da predominância feminina em cursos de turismo, o mercado nem sempre reflete essas distribuições de sexos. De acordo com o Sistema de Informações sobre o Mercado de Trabalho no Setor Turismo (SIMT), na caracterização da ocupação formal no emprego no turismo - ano base de 2018, o mercado era composto por 561.968 trabalhadores e 476.309 trabalhadoras, ou seja, 54, $1 \%$ de homens e 45,9\% de mulheres. Dados do Observatório do Turismo de Minas Gerais (2020) também apontam que o mercado formal de turismo no estado apresenta 49,4\% de trabalhadoras, com o total de 186.087 mulheres e 190.856 homens. Os dados também distinguem que, enquanto a remuneração média das mulheres é de 1.389,60 reais, a dos homens é 1,4 vezes superior, com média de 1.911,15 reais.

Tais dados corroboram com os resultados encontrados por Silveira et al. (2020) que afirmam que os homens recebem, em média, $22 \%$ a mais do que as mulheres no setor. Ademais, há uma segregação no setor turístico, em que homens ocupam as camadas com os maiores salários, apesar das mulheres do setor apresentarem níveis de educação um pouco superior do que homens (Guimarães \& Silva, 2016).

Um dos principais setores de atuação de turismólogas e turismólogos é a docência, e houve ascensão da docência enquanto área de atuação de profissionais egressos dos cursos superiores em turismo entre 2012 e 2018, representando um a cada quatro turismólogos formados (Silveira et al., 2020)incluindo grupos de trabalho em muitos eventos, há, do outro lado, pouca informação acerca da empregabilidade dos egressos desses cursos. Houve ao longo do tempo um distanciamento notório entre os inte- resses dos formadores e dos contratantes, sendo os primeiros mais voltados à construção acadêmica e humanística dos graduandos e os últimos com foco em profissionais com ha- bilidades adquiridas de forma adequada durante seu período de formação. A partir da com- paração entre os dados da atuação profissional dos egressos de cursos superiores em tu- rismo 2012 e 2018, o presente artigo discute o mercado de trabalho de egressos de cursos superiores no Brasil, no que diz respeito às principais atividades, setores de atuação e re- muneração. Baseia-se em dados primários e secundários, com caráter descritivo e enfoque qualitativo na interpretação dos dados. Entre os principais resultados destacam-se que o mercado de trabalho em turismo não é tão diversificado quanto se pensa e se ensina nos cursos de turismo já que se percebe concentração de turismólogos em uma única ou poucas áreas de atuação; uma percepção da melhora discreta na remuneração e aumento da par- ticipação de docentes no mercado e o aumento de respondentes ligados ao setor público" (Silveira et al., 
2020). Nesse sentido, observa-se a relevância de compreender a representatividade feminina também nesta área de atuação. A literatura anterior aponta para diferenças na proporção entre homens e mulheres na academia em geral. No Brasil, mais de $40 \%$ dos pesquisadores entre 2011-2015 eram do sexo feminino, mas há grande variação conforme a área de pesquisa, com maior quantitativo feminino nas áreas relativas à saúde e vida (Allagnat et al., 2017). Contudo, sabe-se que existem fatores que interferem na carreira conforme o gênero, como a criação filhos e relacionamento com o cônjuge, fazendo com que mulheres tenham maior chance de desistir da docência (Goulden et al., 2011). Ademais, não existem estudos sobre a atuação de docentes nos PPGTurs brasileiros que enfatizem uma análise por sexo (TurisData UNIRIO, n.d.).

Assim, o objetivo deste artigo é compreender como a inserção feminina de docentes de turismo está acontecendo no âmbito da Associação Nacional de Pesquisa e Pós-Graduação em Turismo (Anptur). A Associação Nacional de Pesquisas e Pós-Graduação em Turismo foi escolhida por representar uma das principais e mais antigas associações de turismo do Brasil, fundada em 2002. Ademais, em 2019, a Anptur criou um grupo de trabalho dedicado aos estudos de gênero e turismo, dentro da divisão científica de sociedade e cultura, reforçando a pertinência das discussões apresentadas nesta pesquisa.

Neste sentido, é necessário ressaltar que, ao abordar a atuação docente na academia, o estudo não tem um enfoque de gênero, e sim, de sexo, uma vez que não há distinção sobre a identidade de gênero dos docentes investigados. Deste modo, o estudo contribui ao apresentar um recorte sobre a atuação docente em programas de pós-graduação em turismo, contribuindo para o entendimento da representatividade e da influência dos diferentes sexos na Anptur.

\section{FUNDAMENTAÇÃO TEÓRICA}

\section{Mulheres na CiÊNCiA}

Estimativas apontam para a diminuição da disparidade de gênero na academia em relação à contratação, angariação de fundos, produtividade e publicações (West et al., 2013). No Brasil, a proporção entre mulheres e homens que publicam em periódicos acadêmicos registrou um aumento de 11 pontos percentuais entre 2011 e 2015, fazendo com que as mulheres constituam 49\% das pesquisadoras no país (Allagnat et al., 2017). Contudo, há variações sobre a equidade de gênero entre as áreas (Allagnat et al., 2017; Bayazit, 2020; Walker \& Webster, 2004) e a existência da disparidade de gênero ocorre de formas sutis, muitas vezes difíceis de capturar por meio de mapeamentos quantitativos (West et al., 2013). Globalmente, em média, mulheres publicam menos artigos do que homens; entretanto, não há evidências em como isto afeta nem a citação nem o download dos artigos (Allagnat et al., 2017). Ainda há predominância de autores de sexo masculino entre os primeiros e últimos autores, bem como uma baixa representatividade de mulheres como autoras de artigos de autoria única (Nunkoo et al., 2020; West et al., 2013). Ademais, mulheres representam a minoria entre inventores e detentores de patentes ao redor do globo (Bayazit, 2020) e elas também têm uma maior probabilidade de desistirem da carreira de pesquisador na transição entre discente e carreira docente (Adamo, 2013; Martinez et al., 2007). Alguns fatores podem contribuir para o entendimento das discrepâncias entre a atuação 
acadêmica de mulheres e homens. As mulheres têm menos probabilidade do que os homens de colaborar internacionalmente em trabalhos de pesquisa (Allagnat et al., 2017). Entre os pesquisadores, as mulheres geralmente têm menos mobilidade internacional do que os homens, sendo que um maior impacto das citações acadêmicas está associado a pesquisadores transitórios, isto é, pesquisadores que se mudam internacionalmente por períodos inferiores a dois anos (Allagnat et al., 2017). Artigos científicos de pesquisadoras tendem a ter menos citações em comparação com aqueles de autoria masculina, e uma explicação pode envolver o fato de homens serem maioria no corpo editorial de periódicos, bem como revisores tenderem a favorecer autores do mesmo sexo (Ramalho et al., 2020).

Portanto, os valores de citações de impacto são mais altos para os homens do que para as mulheres, principalmente no Brasil, Portugal, México e Chile (Allagnat et al., 2017) Fatores familiares também são mais importantes para mulheres do que homens, sendo que a formação familiar representa um dos maiores vazamentos entre a conclusão do doutorado e a conquista de estabilidade para mulheres nas ciências (Goulden et al., 2011). Mulheres fazem mais concessões, e acham mais importante do que os homens, ter filhos, passar tempo com os filhos e passar tempo com a família (Martinez et al., 2007). Adamo (2013) reporta que o período de maior competitividade na academia acontece no período de busca por posições na academia, período no qual mais mulheres tendem a ter parceiros, assim como filhos. Isso também impacta na necessidade de mais mulheres do que homens importarem-se com creches econômicas, horas de trabalho flexíveis, trabalhos de meio período e emprego perto do esposo (Martinez et al., 2007).

Outros fatores que interferem nessa transição entre o doutoramento e a vida acadêmica são experiências negativas como aluno de doutorado, outros interesses de vida, atividades profissionais que consomem muito tempo, questões relacionadas a filhos e questões de localização geográfica (Goulden et al., 2011).

Como soluções, instituições acadêmicas deveriam promover interações transinstituicionais entre pós-doutorandos e pesquisadores seniores, possibilitar mentorias, treinamentos e considerar as necessidades particulares das mulheres (Martinez et al., 2007). Goulden et al. (2011: 158-159) acrescentam que instituições de pesquisa e universidades devem: 1) promover políticas de resposta à família que sejam básicas, claras e bem-comunicadas para todas as classes de pesquisadores; 2 ) prover suplementos financeiros adicionais para compensar a perda de produtividade por questões familiares; 3 ) mover-se de forma colaborativa em direção a um pacote completo de políticas e recursos favoráveis à família que levam em consideração o curso de vida profissional e familiar; 4) remover os critérios baseados no tempo para bolsas e avaliações de produtividade que não reconheçam eventos familiares e seu impacto no tempo de carreira; 5) coletar e analisar os dados necessários para garantir que as iniciativas políticas existentes, e futuras, sejam eficazes para atender às necessidades dos pesquisadores. Nesse sentido, instituições de pesquisa precisam ficar atentas às questões relacionadas à inequidade de gênero e tomar medidas que promovam ações mais igualitárias. 


\section{TURISMO E REPRESENTATIVIDADE FEMININA NO BRASIL}

No Brasil, de acordo com Ribeiro (2018), o movimento feminista começou ainda no século XIX com reinvidicações relacionadas ao direito ao voto e à vida pública. Em 1922 surge a Federação Brasileira pelo Progresso Feminino com o objetivo de lutar pelo sufrágio feminino e pelo direito ao trabalho sem a necessidade de autorização do marido, esses primeiros acontecimentos são conhecidos como a primeira onda do feminismo no País. A segunda onda do feminismo começou na década de 1970 com o objetivo de lutar pela valorização do trabalho, pelo direito ao prazer e contra a violência sexual. De acordo com Heilborn e Sorj (1999), os estudos desse período no Brasil começam principalmente dentro das universidades e liderado por acadêmicas. Os estudos feministas ganharam maior visibilidade a partir de 1975 sob as denominações "estudos sobre a mulher", "estudos de gênero" e "relações de gênero". Tais termos evoluiram ao longo dos anos, a partir do desenvolvimento do conhecimento. Na década de setenta os "Estudos sobre a Mulher" surgiram para preencher lacunas sobre a situação das mulheres na sociedade. A partir da década de oitenta, então, há a substituição para o termo "gênero". Esta mudança favoreceu a rejeição do determinismo biológico na construção da compreensão sobre o feminino e o marculino.

A terceira onda do feminismo, então, é iniciada na década de 1990 e foi alavancada por Judith Butler (Ribeiro, 2018). Neste momento a crítica se dá contra o discurso universal sobre o que é ser mulher e a maior crítica é ao sistema binário homem/mulher. Aprofunda-se, portanto, nas diferenças entre sexo e gênero e suas relações e opressões (Butler, 2019). Desde então, os estudos de gênero se desenvolvem nas diversas áreas de conhecimento, incluindo o turismo.

A discussão de gênero se trata da base para a mudança e potencial equidade entre homens e mulheres (Swain, 1995). Tal discussão no turismo não é recente, mas Allagnat et al. (2017) apontam um aumento no número de pesquisas sobre o tema. Swain (1995) se refere a gênero como um sistema de identidades culturais e relações sociais entre homens e mulheres, sendo distinto de diferenças biológicas entre os sexos. O gênero é visto na sociologia com uma variável importante que interfere no comportamento e nas oportunidades, já que mulheres e homens se envolvem de maneiras diferentes na construção e consumo do turismo (Brown \& Osman, 2017).

Para se entender gênero e turismo há três problemas que são considerados centrais, conforme Swain (1995):

1. Processos turísticos são construídos na sociedade a partir de relações de gênero;

2. Relações de gênero ao longo do tempo estão interconectadas com dimensões da sociedade comprometidas com práticas de turismo, tais quais: ambiente, cultura, sociais, econômicas e políticas;

3. Problemas referentes a poder, controle e equidade são articulados através de relações de gênero, raça e classe no turismo.

É relevante destacar que esta pesquisa tem o objetivo, conforme apresentado, de analisar a representatividade do sexo feminino nos contextos estudados, mas não possui um enfoque de gênero. Para tanto, apoia-se em Butler (2019), que afirma que, enquanto o sexo é 
reconhecido dentro de uma estrutura binária biológica mulher/homem, o desenvolvimento do gênero se dá por meio de diversas e complexas influências culturais e também de compreensão dos corpos sexuais e, portanto, envolvem uma diversidade de identidades de gênero possíveis. Este estudo, portanto, de acordo com o seu objetivo e procedimentos metodológicos - que serão apresentados na próxima seção -, optou por estudar a representatividade das mulheres no turismo a partir da distinção de sexos.

\section{METODOLOGIA}

A fim de explorar a representatividade e a influência feminina na Anptur, o estudo foi baseado em um levantamento quantitativo com base em dados secundários considerando a composição por sexo das seguintes variáveis de análise:

a) docentes dos nove Programas de Pós-Graduação em Turismo vinculados à Anptur (Universidade Anhembi Morumbi (UAM), Universidade Caxias do Sul (UCS), Universidade Estadual do Ceará (UECE), Universidade Federal Fluminense (UFF), Universidade Federal de Pernambuco (UFPE), Universidade Federal do Paraná (UFPR), Universidade Federal do Rio Grande do Norte (UFRN), Universidade do Vale do Itajaí (Univali) e Universidade de São Paulo (USP);

b) autores dos trabalhos apresentados na Anptur;

c) premiações concedidas pela Anptur;

d) diretoria da Anptur;

e) citações dos trabalhos publicados por esses docentes.

Os dados de docentes foram coletados nos sites dos PPGTurs disponíveis na internet. Já os dados de citações foram coletados dos perfis públicos desses mesmos docentes no Google Scholar na primeira semana de junho de 2020 (citações totais; citações desde 2015; h-index; i-index desde 2015). As informações sobre os prêmios distribuídos e a composição da diretoria foram solicitados diretamente à secretaria da entidade, que forneceu os dados disponíveis nos registros existentes (premiações de 2007 a 2019; diretoria de 2011 a 2020).

Os(as) autores(as) dos trabalhos apresentados na Anptur foram coletados diretamente nos Anais de 2005 a 2019. No momento de análise dos dados, a base de dados dos Anais do Seminário da Anptur agregava 5.327 trabalhos publicados e 2.496 autores(as). O sexo dos(as) autores(as) foi imputado a partir do nome próprio, utilizando a linguagem de programação em Python, Gender.py, disponibilizada pelo API Genderize.io e processada no ambiente Visual Studio Code (VSCode). Genderize.io possui uma base de dados com 114.541.298 nomes coletados e baseados na autodeclaração de sexo das redes sociais de diferentes nacionalidades. Para o processamento foi realizada a organização para que em cada lista constasse somente o primeiro nome dos indivíduos. A partir disso, para cada nome próprio foi fornecida uma previsão de sexo (feminino/masculino), bem como uma estimativa de probabilidade para a previsão, para a qual foi aceita a certeza de atribuição acima de 0,99. Para casos de resultado desconhecido, a lista foi reprocessada e, em último caso, foi realizada a conferência e atribuição manual da informação de sexo. 
A análise dos dados se deu com base na estatística descritiva (Hair et al., 2005) com suporte do software SPSS, buscando-se comparar a distribuição por sexo em todas as variáveis definidas no estudo.

\section{RESULTADOS}

Os resultados são apresentados em 4 tópicos distintos. Primeiro, apresenta-se a representatividade docente nos programas de pós-graduação em turismo vinculados à Anptur. Em um segundo momento, demonstra-se a representatividade por meio de um levantamento da autoria de trabalhos apresentados nos Seminários da Anptur entre 2005 e 2019. Em seguida, são analisados os prêmios e a composição da diretoria da Anptur. Por fim, avaliam-se as citações dos docentes com base nas informações do Google Scholar.

\section{Representatividade Docente nos Programas de Pós-Graduação em Turismo vinculados à ANPTUR}

O levantamento realizado nos sites dos PPGTurs mostrou que, em 2020, existiam 128 docentes ativos nos nove programas vinculados à Anptur. Dentre esses, 60 (47\%) são do sexo feminino e 68 (53\%) são do sexo masculino (Figura 1).

Figura 1 - Docentes de PPGTURs vinculados à Anptur, por sexo.

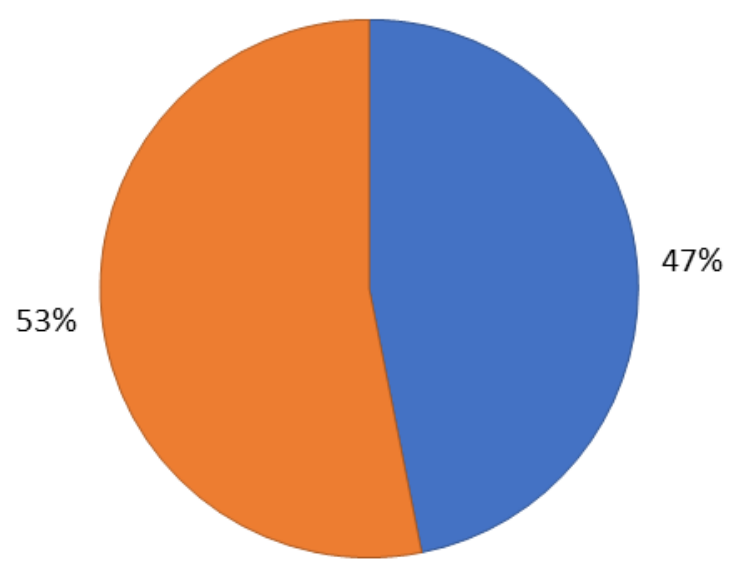

- Feminino $\square$ Masculino

Fonte: Autoras.

Observa-se que a distribuição por sexo varia entre os PPGTurs (Tabela 1), sendo que quatro programas (UAM, UCS, UECE e UFPE) contam com maioria de docentes do sexo feminino e cinco programas contam com predominância do sexo masculino (UFF, UFPR, UFRN, UNIVALI e USP). 
Tabela 1- Representatividade Docente nos PPGTurs vinculados à Anptur, por sexo.

\begin{tabular}{|c|c|c|c|c|}
\hline \multicolumn{5}{|c|}{ Representatividade Docente por Sexo nos PPGTurs } \\
\hline Instituição/ Sexo & Feminino & Masculino & Feminino (\%) & Masculino (\%) \\
\hline UAM & 6 & 3 & 66,67 & 33,33 \\
\hline UCS & 10 & 5 & 66,67 & 33,33 \\
\hline UECE & 7 & 6 & 53,85 & 46,15 \\
\hline UFF & 8 & 9 & 47,06 & 52,94 \\
\hline UFPE & 8 & 5 & 61,54 & 38,46 \\
\hline UFPR & 6 & 7 & 46,15 & 53,85 \\
\hline UFRN & 7 & 9 & 43,75 & 56,25 \\
\hline UNIVALI & 6 & 10 & 37,50 & 62,50 \\
\hline USP & 2 & 14 & 12,50 & 87,50 \\
\hline Total & 60 & 68 & $47 \%$ & $53 \%$ \\
\hline
\end{tabular}

Fonte: Autoras

As maiores desigualdades entre sexos foram encontradas nos programas da USP e UNIVALI, com menor presença do sexo feminino, e nos programas da UAM, UCS e UFPE, onde é menor a parcela de docentes do sexo masculino. O programa da USP é o que apresenta a maior discrepância, com apenas duas docentes vinculadas.

Figura 2 - Percentagem de docentes vinculados a Programas de Pós-Graduação em Turismo vinculados à Anptur, por sexo.

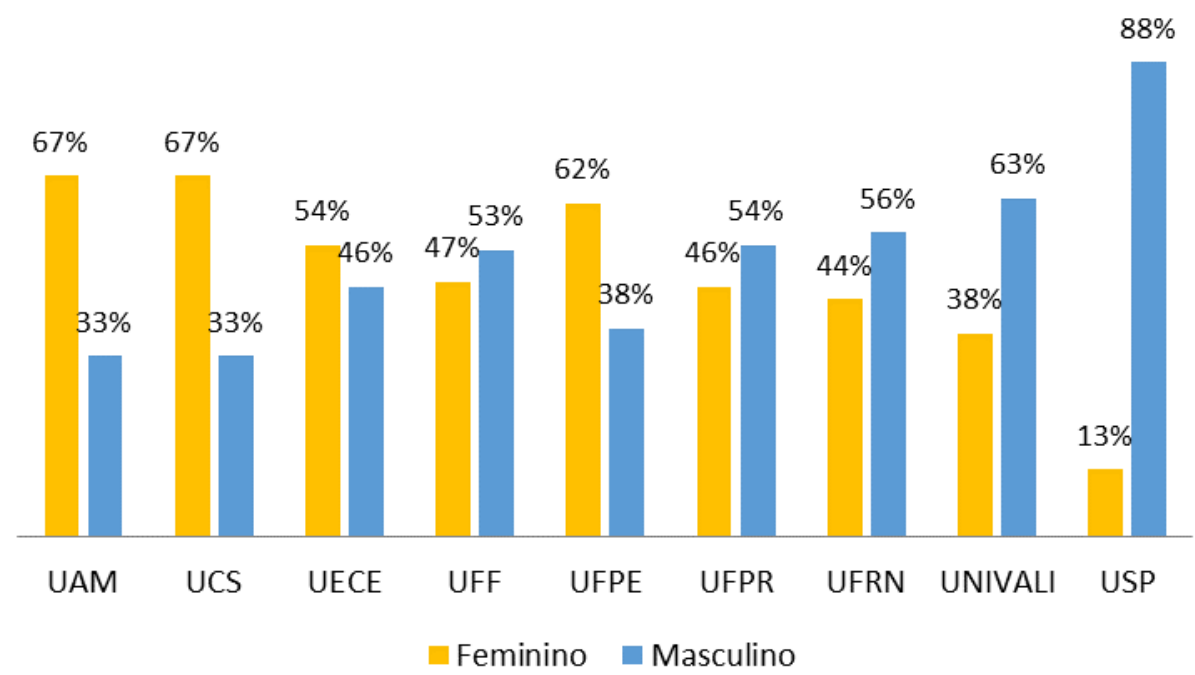

Fonte: Autoras.

Sobre a atuação em cargos de coordenação dos nove Programas de Pós-Graduação em Turismo, tem-se que há três docentes do sexo feminino ocupando cargos de coordenação, sendo duas em programas privados. De outra forma, há seis coordenadores do sexo masculino e três do sexo feminino. Também se observa que há docentes do sexo feminino ocupando cargos de vice coordenação (3 docentes do sexo feminino e três docentes do sexo masculino). 
Tabela 2 - Cargos de Coordenação nos PPGTURs, vinculados à Anptur, por sexo.

\begin{tabular}{|c|c|c|}
\hline Cargos de Coordenação & Coordenador (a) & Vice ou Outra coordenação \\
\hline UAM & Feminino & - \\
\hline UCS & Feminino & - \\
\hline UECE & Masculino & Feminino \\
\hline UFF & Masculino & - \\
\hline UFPE & Masculino & Feminino \\
\hline UFPR & Masculino & Feminino \\
\hline UFRN & Feminino & Masculino \\
\hline UNIVALI & Masculino & Masculino \\
\hline USP & Masculino & Masculino \\
\hline
\end{tabular}

Fonte: Autoras.

Se avaliado o conjunto dos cargos de liderança nos PPG (coordenação e vice), há nove docentes do sexo masculino (60\%) e seis docentes do sexo feminino (40\%).

Esses resultados precisam ser analisados em perspectiva. Apesar do sexo feminino ser predominante nos cursos de graduação e pós-graduação em turismo (Minasi \& Censon, 2020), a carreira docente na área não acompanha essa proporção, em geral. Alguns fatores podem influenciar esse cenário, como a multidisciplinaridade do turismo, com docentes vindos de áreas de conhecimento diversas, o perfil dos programas, ou ainda das dificuldades enfrentadas pelas mulheres para ingressarem e se manterem na carreira docente, entre elas, as questões familiares e a dificuldade das mulheres de saírem do contexto discente e ingressarem em cargos docentes (Goulden et al., 2011).

\section{Autoria de Trabalhos Apresentados nos Seminários da Anptur}

Nos anais dos Seminários da Anptur de 2005 a 2019 foram publicados 2.496 trabalhos por 5.327 autores. Nas publicações, existe uma clara predominância de autorias do sexo feminino (Figura 3).

Figura 3 - Autoria de trabalhos publicados nos anais da Anptur entre 2005 a 2019, por sexo.

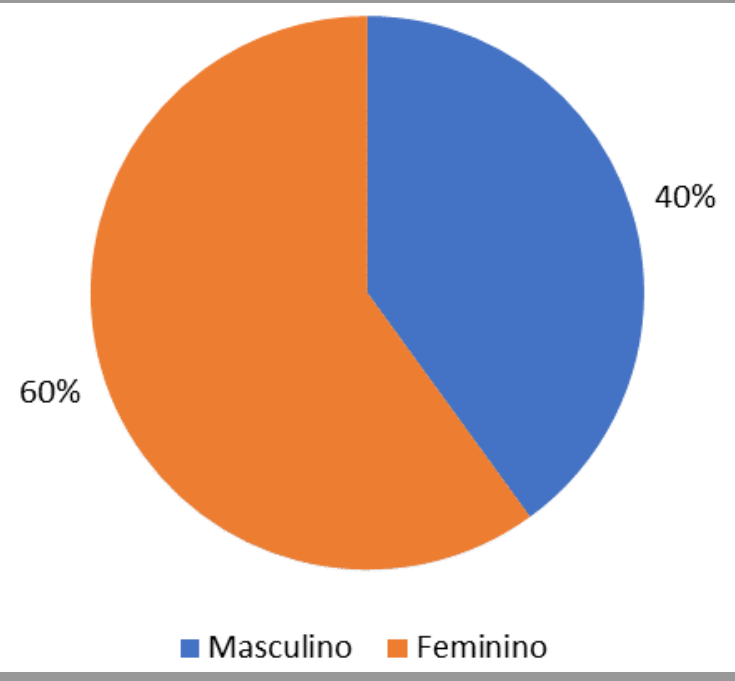

Fonte: Anais Anptur 2005-2019. 
Observando-se a série histórica dos trabalhos (Figura 4), é possível verificar que a autoria do sexo feminino sempre foi em maior número do que do sexo masculino nos últimos 15 anos.

Figura 4 - Percentagem de autores em trabalhos publicados nos anais da Anptur entre 2005 e 2019 , por sexo.

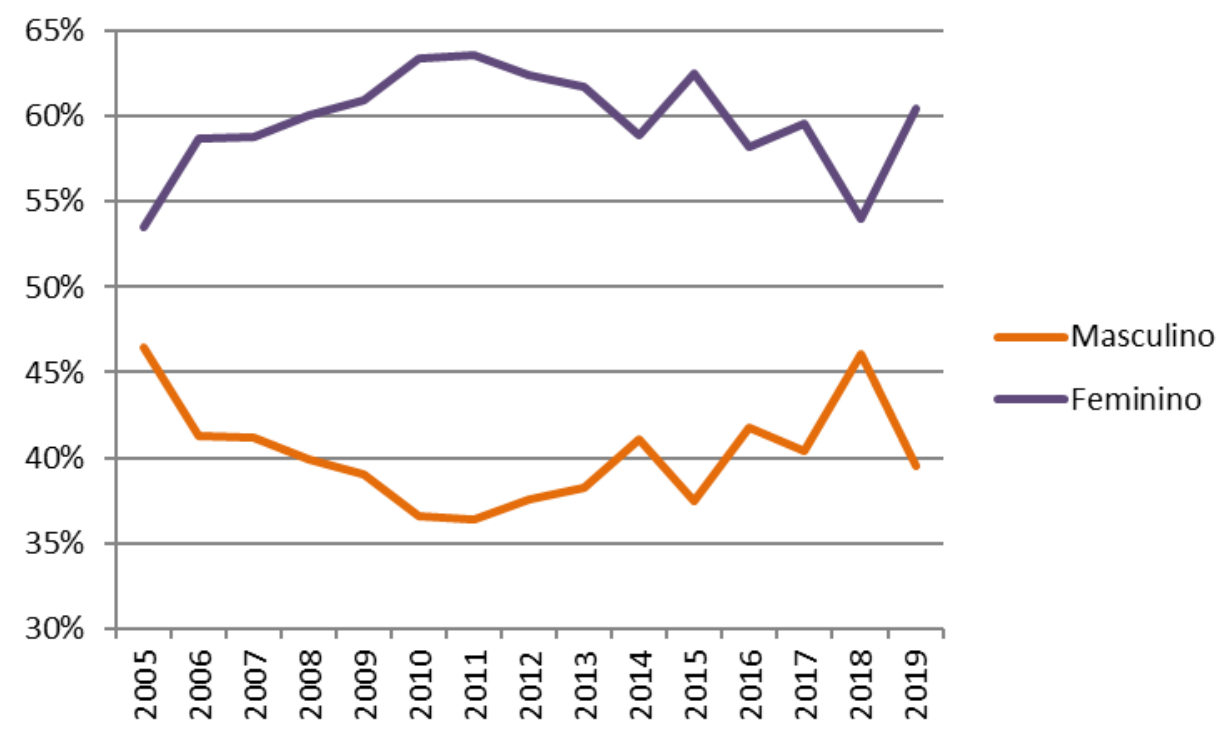

Fonte: Anais Anptur, 2005 a 2019.

Esses dados corroboram a literatura existente, mostrando que, apesar da predominância de mulheres em trabalhos da Anptur, há um descompasso com o avanço para a carreira acadêmica e para espaços de influência na academia do turismo. Isto pode ocorrer em razão da maior probabilidade de mulheres abandonarem a vida acadêmica, cedendo a demandas familiares (Goulden et al., 2011).

\section{PRÊMIOS E COMPOSIÇÃO DA DIRETORIA DA ANPTUR}

Se considerados os três prêmios em conjunto promovidos pela Anptur desde 2007, sendo eles: Pesquisador Destaque, Pesquisador Emérito e Jovem Pesquisador, há um número equilibrado de prêmios concedidos a docentes do sexo feminino (dez) e masculino (dez). De forma específica, entre os pesquisadores destaque, oito dos premiados são do sexo feminino e cinco do sexo masculino. Dentre os pesquisadores eméritos, quatro são do sexo masculino e dois são do sexo feminino. Apenas um jovem pesquisador foi premiado, sendo ele, do sexo masculino (Tabela 3). 
Tur., Visão e Ação, v23, n3, p595-615, Set./Dez. 2021 - Balneário Camboriú, Santa Catarina, Brasil

Tabela 3 - Premiações da Anptur entre 2007 a 2019, por sexo

\begin{tabular}{|c|c|}
\hline Pesquisador Destaque Anptur & Prêmio Pesquisador Emérito \\
\hline 2007 - Masculino & 2010 - Masculino \\
\hline 2008 - Feminino & 2013 - Feminino \\
\hline 2009 - Feminino & 2014 - Masculino \\
\hline 2010 - Feminino & 2016 - Masculino \\
\hline 2011 - Masculino & 2017 - Feminino \\
\hline 2012 - Feminino & 2018 - Masculino \\
\hline 2013 - Feminino & \\
\hline 2014- Masculino & Jovem Pesquisador \\
\hline 2015- Feminino & \multirow{5}{*}{2010 - Masculino } \\
\hline 2016-Masculino & \\
\hline 2017 - Feminino & \\
\hline 2018 - Feminino & \\
\hline 2019 - Masculino & \\
\hline
\end{tabular}

Fonte: Dados cedidos pela Secretaria da Anptur.

Por fim, avaliou-se a representatividade nos cargos de diretoria da Anptur. Existiram quatro diretorias durante o período informado pela secretaria da entidade, de 2011 e 2019. Nesse período, participaram da diretoria oito docentes do sexo feminino e treze docentes do sexo masculino. Nos últimos 9 anos, 8 docentes do sexo feminino ocuparam 19 cargos de gestão nas diretorias da Anptur, e 13 docentes do sexo masculino ocuparam 24 cargos no mesmo período.

Tabela 4 - Participação em cargos de Diretorias da Anptur entre 2011 - 2020, por sexo.

\begin{tabular}{|c|c|c|}
\hline 2011-2020 & $\begin{array}{c}\text { Aparece em mais de uma } \\
\text { gestão }\end{array}$ & Sem repetição \\
\hline Feminino & 19 & 8 \\
\hline Masculino & 24 & 13 \\
\hline Total & 43 & 21 \\
\hline
\end{tabular}

Fonte: Dados cedidos pela Secretaria da Anptur.

Considerando-se que o papel de uma entidade acadêmica deva ser o de abrir espaços para discussões relevantes, agir politicamente e lutar para ampliar espaços onde talentos possam florescer, independentemente de sexo ou gênero, os dados aqui apresentados mostram que a Anptur tem feito um esforço para reconhecer, premiar e dar voz a lideranças femininas, mesmo que este ainda seja um movimento tímido e com poucos resultados visíveis em curto prazo.

Também há de se destacar que, em 2019, o Seminário da Anptur, principal evento nacional da área, criou um grupo de trabalho (GT) de estudos de gênero em turismo no qual foram apresentadas pesquisas sobre as seguintes temáticas: relações sociais de gênero e mercado turístico; parada do orgulho LGBT de São Paulo; a experiência de mulheres que viajam sozinhas; e abordagens teórico práticas de estudos de gênero e turismo. Este GT inaugura um espaço destinado exclusivamente para o tema no principal evento de turismo nacional e aponta para a importância de aprofundar-se nas questões de gênero e da mulher no turismo, sobre a qual este estudo se apoia. 


\section{Citações dos Docentes de Pós-Graduação em Turismo vinculados À} ANPTUR

A influência de pesquisadoras e pesquisadores em um campo de conhecimento pode ser medida pela quantidade de vezes que seus trabalhos são citados. No Brasil, onde bases estatísticas confiáveis sobre citações na área de Turismo são limitadas, uma forma de verificar o número de citações dos docentes dos PPGTurs é por meio dos perfis públicos do Google Scholar. Ao criarem o seu perfil, docentes passam a contar com a busca automática do Scholar de suas produções, como livros e artigos, e dos trabalhos que venham a citá-los, como trabalhos de conclusão de curso, dissertações, teses, artigos, entre outros. Há uma certa margem de erro na ferramenta, mas ela tem sido cada vez mais aceita e está entre as plataformas atualmente consideradas pela CAPES para a avaliação do desempenho dos PPGTurs. No levantamento realizado, dos 128 docentes ativos nos programas vinculados à Anptur, foram encontrados 70 docentes (55\%) com perfis públicos no Scholar, sendo 42 do sexo feminino (60\% do total) e 28 do sexo masculino ( $40 \%$ do total).

O Scholar oferece alguns indicadores, dentre eles o número total de citações das obras de docentes e o h-index, que indica o número de artigos com citações maiores ou iguais a esse número - uma medida que permite quantificar a produtividade e o impacto de cientistas tendo como base seus artigos mais citados. Por exemplo, um pesquisador com $\mathrm{h}=3$, tem 3 artigos que receberam 3 ou mais citações. Esses mesmos indicadores também estão disponíveis desde 2015, permitindo verificar tendências relativas à importância crescente ou decrescente dos trabalhos publicados. Após a coleta nos perfis individuais, foram calculadas algumas medidas descritivas gerais e relacionadas a cada sexo.

As médias gerais de desempenho acadêmico dos docentes dos PPGTurs são: 326,2 citações; 180,9 citações a partir de 2015; h-index de 7,6 e h-index a partir de 2015 de 6,0. No entanto, as médias precisam ser consideradas com cuidado, uma vez que a distribuição dos dados de docentes dos PPGTurs apresenta uma assimetria positiva, com uma grande concentração de pesquisadores com poucas citações e baixa influência acadêmica. Por exemplo, a maioria dos docentes ainda não atingiu a marca das 100 citações (37\%), uma pequena minoria (7\%) superou o número de 1.000 citações, com apenas um docente acima de 3.000 citações. Trata-se, portanto, de uma área ainda em desenvolvimento e dominada pela influência de poucos pesquisadores.

Observando-se os resultados por sexo discriminados na Tabela 5, verifica-se que o sexo masculino supera, em média, o feminino em todos os indicadores. O valor mínimo de citações entre os sexos é similar, mas quando analisamos os valores máximos, há uma grande diferença. Mesmo nos indicadores que refletem os últimos cinco anos, a predominância da influência masculina parece se confirmar e se manter como tendência. 
Tur., Visão e Ação, v23, n3, p595-615, Set./Dez. 2021 - Balneário Camboriú, Santa Catarina, Brasil

Tabela 5 - Citações dos Docentes dos PPGTurs, por sexo.

\begin{tabular}{|c|c|c|c|c|c|}
\hline \multicolumn{6}{|c|}{ Citações } \\
\hline \multirow{5}{*}{ Feminino } & Média & 282,7 & \multirow{5}{*}{ Masculino } & Média & 357,8 \\
\hline & Mediana & 139,0 & & Mediana & 171,0 \\
\hline & Desvio Padrão & 387,9 & & Desvio Padrão & 599,7 \\
\hline & Mínimo & 16,0 & & Mínimo & 15,0 \\
\hline & Máximo & 1854,0 & & Máximo & 3059,0 \\
\hline \multicolumn{6}{|c|}{ Citações a partir de 2015} \\
\hline \multirow{5}{*}{ Feminino } & Média & 148,9 & \multirow{5}{*}{ Masculino } & Média & 204,2 \\
\hline & Mediana & 82,0 & & Mediana & 120,0 \\
\hline & Desvio Padrão & 153,9 & & Desvio Padrão & 284,0 \\
\hline & Mínimo & 11,0 & & Mínimo & 12,0 \\
\hline & Máximo & 760,0 & & Máximo & 1630,0 \\
\hline \multicolumn{6}{|c|}{ h-index } \\
\hline \multirow{5}{*}{ Feminino } & Média & 7,3 & \multirow{5}{*}{ Masculino } & Média & 7,9 \\
\hline & Mediana & 6,0 & & Mediana & 7,0 \\
\hline & Desvio Padrão & 3,9 & & Desvio Padrão & 4,6 \\
\hline & Mínimo & 2,0 & & Mínimo & 2,0 \\
\hline & Máximo & 19,0 & & Máximo & 27,0 \\
\hline \multicolumn{6}{|c|}{ h-index a partir de 2015} \\
\hline \multirow{5}{*}{ Feminino } & Média & 5,6 & \multirow{5}{*}{ Masculino } & Média & 6,3 \\
\hline & Mediana & 5,0 & & Mediana & 6,0 \\
\hline & Desvio Padrão & 2,2 & & Desvio Padrão & 3,4 \\
\hline & Mínimo & 2,0 & & Mínimo & 2,0 \\
\hline & Máximo & 12,0 & & Máximo & 20,0 \\
\hline
\end{tabular}

Fonte: Saída SPSS, com base nos dados extraídos do Google Scholar.

Como havia uma quantidade menor de docentes do sexo feminino na amostra, devido à limitação de acesso aos perfis do Scholar, e considerando-se a grande concentração de citações em poucos docentes, foram também avaliadas as produções dos 10 docentes com maior quantidade de citações. A análise revelou que existem 4 mulheres entre os 10 docentes mais influentes dos PPGTurs, ocupando a quarta, sexta, sétima e nona posições em quantidade total de citações.

Figura 5- Top 10 de Pesquisadores de Turismo mais citados no Google Scholar

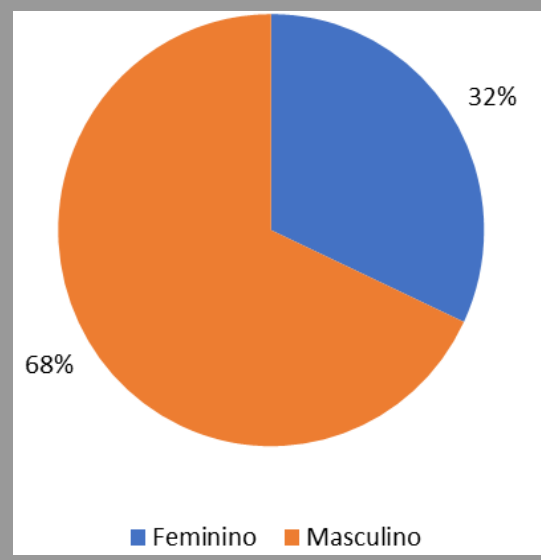

Fonte: Baseado em dados do Google Scholar. 
Com relação à quantidade de citações a partir de 2015, essas mesmas docentes caem um pouco de posição, ocupando a quinta, sétima, oitava e décima posições. Em suma, entre docentes influentes, os do sexo masculino mantém uma vantagem ainda maior em relação às do sexo feminino (Figuras 5 e 6 ).

Figura 6 - Top 10 de Pesquisadores de Turismo mais citados no Scholar Google desde 2015

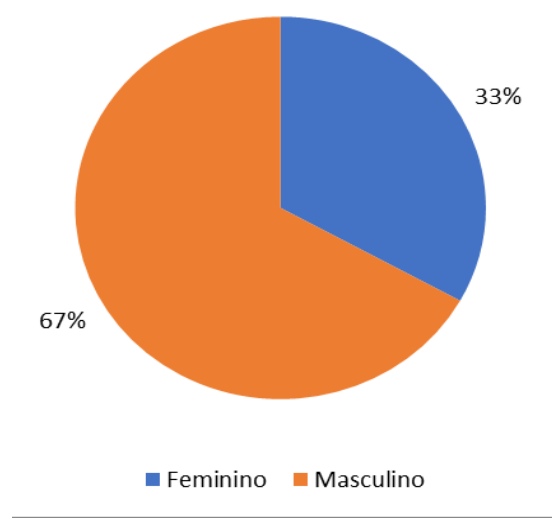

Fonte: Baseado em dados do Google Scholar.

É preciso reconhecer que, entre os docentes mais influentes dos PPGTurs, estão pesquisadores que atuam há muito tempo na área. Infelizmente, o Scholar não oferece uma análise das citações de acordo com os tipos de documentos. Por exemplo, não há indicadores específicos para o impacto de livros e de artigos científicos. Isto seria importante para que se identificassem vieses nos dados, como docentes que acumularam muitas citações ao longo dos anos devido à publicação de livros didáticos adotados em cursos de turismo, especialmente aqueles publicados nos anos em que houve um grande crescimento de cursos de graduação dedicados a área em todo o Brasil. Parece ser baixa a influência de pesquisadores de ambos os sexos com carreiras em desenvolvimento, dedicados a publicar em revistas científicas nacionais e internacionais, sendo difícil saber se há diferenças entre os sexos neste quesito.

Analisando-se a distribuição dos dados sem os docentes outliers (Figuras 7 e 8) é possível observar que, nos indicadores de citações a partir de 2015, os docentes do sexo masculino mantêm a tendência de um desempenho acadêmico superior. 
Figura 7 - Boxplot de citações de pesquisadores de turismo no Google Scholar desde 2015, por sexo, incluindo outliers.

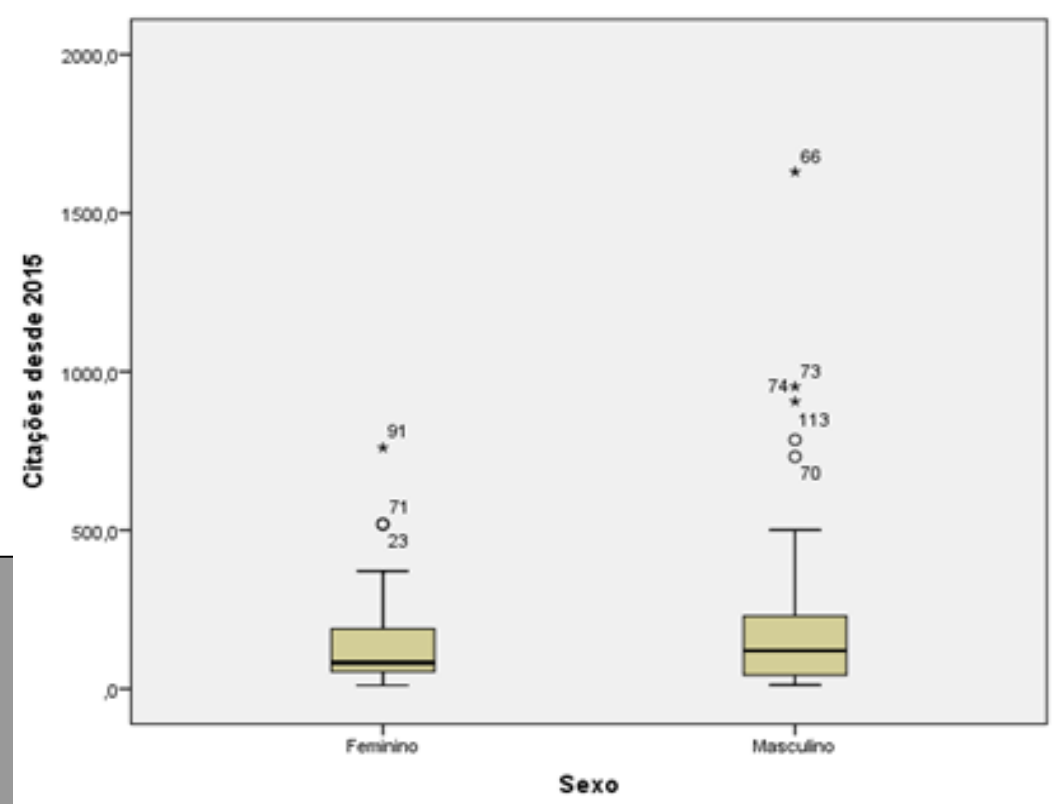

Fonte: Saída SPSS.

Estes resultados mostram que, entre docentes dos PPGTurs, excetuando-se aqueles poucos com grande influência na área, as do sexo feminino estão mais concentradas nas faixas baixas de citações do que os do sexo masculino, que têm alcançado melhores resultados acadêmicos.

Figura 8 - Boxplot de h-index de citações de pesquisadores de turismo, por sexo, incluindo outliers.

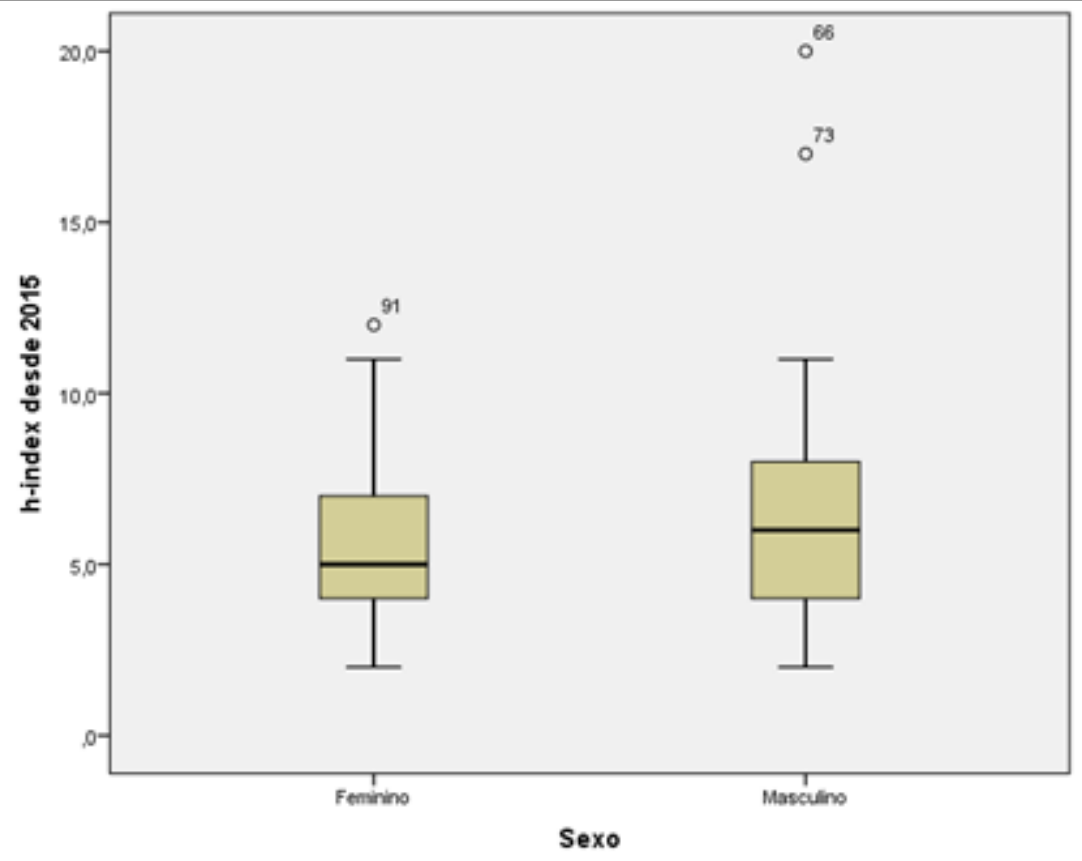

Fonte: Saída SPSS. 
Este cenário reproduz o que outros estudos dessa natureza já mostraram em outras áreas do conhecimento. Em artigos científicos, por exemplo, pesquisadoras tendem a ter menos citações do que pesquisadores (Ramalho et al., 2020)technology, engineering, and math (STEM. As mulheres têm menor probabilidade do que os homens de colaborar internacionalmente em trabalhos de pesquisa (Allagnat et al., 2017), têm menos mobilidade internacional do que os homens, sendo que um maior impacto das citações acadêmicas está diretamente associado a esses dois fatores (Allagnat et al., 2017. É possível que existam, ainda, uma série de fatores socioculturais contribuindo para este cenário, uma vez que o Brasil é um dos países que tem maior disparidade na proporção de citações entre homens e mulheres no mundo (Allagnat et al., 2017). Deste modo, o artigo demonstra que, em linhas gerais, as mulheres têm participado como autoras no seminário Anptur, há docentes do sexo feminino atuando nos PPGturs, assumindo alguns cargos de diretoria na Anptur e sendo premiadas pela instituição. Porém, a citação de autores do sexo masculino é especialmente superior à de autoras do sexo feminino na área, se usado como base o Google Scholar. Nesse sentido, deve-se continuar as discussões sobre o tema, a fim de se aprofundar as causas das diferenças de gênero e desafios enfrentados pelas mulheres na academia e no setor de turismo.

A demanda familiar implica em longas horas de trabalho doméstico, comprometendo a imersão e concentração necessárias ao trabalho intelectual e ao amadurecimento de pesquisas inovadoras e de alta qualidade acadêmica. Homens contam, como padrão, com uma rede de apoio feminina que tem impacto direto no avanço de carreiras profissionais (Vaz, 2013). São esposas, irmãs, mães, avós, secretárias, assistentes, faxineiras, cozinheiras, enfermeiras, entre muitas outras mulheres. Essa rede apoia principalmente os homens, mas algumas mulheres também se beneficiam - o que não é fácil ou considerado normal. Uma parcela pequena tem apoio masculino, já que, muitas vezes, a mulher é considerada responsável pelas tarefas domésticas e familiares (Schaap \& Shockley, 2020). Assim, a produção intelectual feminina varia de acordo com o ciclo de criação dos filhos, uma tarefa social fundamental e ainda muito subestimada.

No processo de vazamento entre os estudos e a profissão, talentos ficam incubados e deixam de se desenvolver de forma plena (Goulden et al., 2011). Uma consequência ainda mais perversa deste cenário se refere à segurança financeira futura de mulheres que, quando perdem seus parceiros, possuem maior risco de queda no padrão de vida ou mesmo risco de manutenção própria e da família. O que acaba perpetuando antigas estruturas de poder, dominação e subserviência, uma vez que essas mulheres dependerão da benevolência masculina para sua sobrevivência e para ter acesso a espaços mais privilegiados.

\section{CONSIDERAÇÕES FINAIS}

Em diversos países, observa-se que há desequilíbrio entre a representatividade feminina e masculina nas mais diversas áreas acadêmicas, e esta realidade não é diferente na academia de turismo no Brasil. Apesar de alguns índices sinalizarem para certa paridade entre o número de docentes do sexo feminino e masculino, como é o caso da pesquisa em questão, na qual observamos que, em muitas das instituições em análise, há proporcionalidade entre os sexos; 
muitas vezes esses dados não reverberam em representatividade feminina em cargos de gestão nas Instituições de Ensino e/ou nas Associações de Classe que integram, assim como na área de influência no que tange a produção científica.

Há certo equilíbrio entre o número de docentes do sexo feminino e do sexo masculino atuando em programas de pós-graduação em turismo estudados, com discrepâncias maiores na USP, com maioria masculina. No entanto, sabe-se que há um maior número de discentes mulheres em turismo desde a graduação até mestrado e doutorado (Minasi \& Censon, 2020). Nesse sentido, é possível que outros fatores - como a dificuldade de ingressar na docência e fatores socioculturais relacionados às demandas da criação de filhos, cuidados com outros familiares e cônjuges - interfiram para que essa proporção se apresente de forma relativamente equilibrada entre docentes do sexo feminino e masculino.

Sobre a autoria de trabalhos publicados em anais do Anptur entre 2005 e 2019, tem-se que há maioria de autoras do sexo feminino (60\%), em detrimento de autores do sexo masculino (40\%). Nesse sentido, outras pesquisas podem aprofundar as discussões sobre as dificuldades para que as mulheres permaneçam no cenário acadêmico do turismo no Brasil, uma vez que há indícios de que parte das discentes abandonam a carreira acadêmica; assim como o mercado de trabalho em turismo.

A média de citação de mulheres é inferior à da citação de homens pertencentes ao corpo docente de programas de pós-graduação em turismo brasileiros estudados em todos os índices existentes na plataforma Google Scholar. Tal resultado merece atenção pelo fato de se tratar de uma tendência que não tem mudado ao longo dos anos. Com isso, os autores da área devem se atentar aos fatores que fazem que com que haja citação de livros e artigos de pesquisadores do sexo masculino de forma mais expressiva do que de pesquisadoras.

A respeito dos cargos de coordenação e vice coordenação dos PPGTurs, que possuem maioria masculina (60\%), a inserção menor das mulheres em cargos de liderança merece atenção. Nesta mesma linha, mais homens (62\%) ocupam cargos de diretoria da Anptur, se considerados docentes em uma das 4 diretorias distintas desde 2011 . Resta investigar os motivos da baixa inserção feminina em cargos de liderança, seja em seu próprio PPGTur ou no envolvimento com a Anptur, fator que pode ser abordado em pesquisas futuras.

Já sobre as premiações promovidas pela Anptur desde 2001 (Pesquisador Destaque, Pesquisador Emérito e Jovem Pesquisador), há um número equilibrado de prêmios concedidos a docentes do sexo feminino (dez) e masculino (dez). Todavia, as docentes do sexo feminino têm maioria em premiações de pesquisador destaque, ao passo que pesquisador emérito e jovem pesquisador apresentam maioria masculina. Desse modo, reconhece-se o esforço da Anptur ao buscar a participação feminina em suas ações, bem como a criação do grupo de trabalho para discutir as questões de gênero (desde 2019). A academia pode se beneficiar ao se fortalecer e dar condições de inserção acadêmica, independente do sexo. Este artigo demonstra que é necessário compreender melhor as disparidades de gênero enfrentadas pelas docentes de turismo. Ações que podem ser adotadas para alterar esse cenário, sobretudo, envolvem: 1) estimular mulheres a assumirem cargos de diretoria, coordenação e corpo editorial de periódicos; 2) proporcionar situações favoráveis para o estabelecimento de parcerias entre os docentes 
e estimular que mulheres também busquem tais parcerias; 3) pensar em formas alternativas à respeito da restrição de mobilidade de alguns pesquisadores, por meio de eventos e participação online, por exemplo; 4) promover treinamentos e mentorias para que os pesquisadores possam conhecer o trabalho de outros pesquisadores e desenvolvam suas carreiras, 5) preocupar-se com a representatividade e participação feminina também na organização dos eventos, como escolha de keynote speakers, membros de mesa redonda, revisores, coordenadores de grupo de trabalho e outros; 6) construir, assim como consolidar, redes de apoio às atividades acadêmicas.

Diversas são as limitações deste estudo, sobretudo, de ordem metodológica. Sobre a seleção do corpo docente, sabe-se que existem outros pesquisadores de turismo no Brasil que não estão vinculados a nenhum dos programas citados, bem como a existência de outros programas de pós-graduação em turismo em áreas como hospitalidade, gastronomia e lazer, que não estão vinculados à Anptur. Outras associações da área também podem ser investigadas futuramente, bem como a atuação no nível de graduação, uma vez que os professores podem atuar em áreas como a administração, letras, patrimônio ou geografia, por exemplo. Ainda, uma das limitações deste estudo que pode ser suprida futuramente é a inserção da variável tempo de atuação para ponderar os resultados estudados. Sobre a participação em cargos de diretoria da Anptur, não foi obtido acesso formal aos dados anteriores às diretorias de 2011.

Outra limitação se dá pelo uso exclusivo do Google Scholar, que depende que os autores se cadastrem e confirmem suas informações devidamente. Pode acontecer desse levantamento não apresentar precisão, uma vez que depende da revisão das publicações pelo próprio autor. Ainda, a base do Google Scholar inclui livros e artigos científicos, de forma que, em termos de tempo, envolve momentos diferentes no turismo enquanto área de pesquisa. Atualmente, os programas de pós-graduação são mais cobrados por publicações de artigos em periódicos de alto impacto do que pela participação em eventos e publicação de livros e capítulos. Em outras palavras, outros formatos e fontes de comparação de atuação docente por sexo são bem-vindos. A análise desenvolvida neste artigo prejudica pesquisadores com temas emergentes ou que se dedicam a temas relevantes, mas específicos, temáticas gerais e mais valorizadas em certos setores ganham maior visibilidade. O turismo é uma área pequena em relação a outras áreas do conhecimento e para os que publicam no Brasil, existe a barreira da língua, que impede que os trabalhos em português sejam lidos e citados em outros países.

Este estudo apresenta diversos caminhos para pesquisas futuras. Umas das possibilidades é continuar as comparações por meio do levantamento da representatividade de autores do sexo feminino e masculino em artigos publicados em periódicos nacionais e internacionais de turismo. Uma hipótese a ser testada em estudos futuros pode ser se docentes com maior tempo de atuação em programas de pós-graduação apresentam um número maior de citações. Avaliar a representatividade do corpo discente dos programas de pós-graduação e graduação em turismo também pode ser um caminho futuro. Ademais, aponta-se a necessidade de pesquisar a inserção de mulheres em cargos de gestão e os motivos que fazem com que existam 'vazamentos' na carreira feminina que as impedem de atuar, seja como docentes ou em cargos de gestão pública e privada da cadeia do turismo. Finalmente, outro tópico de pesquisa que merece atenção é a produtividade feminina durante a pandemia do COVID19. 


\section{REFERÊNCIAS}

Adamo, S. A. (2013). Attrition of women in the biological sciences: Workload, motherhood, and other explanations revisited. BioScience, 63(1), 43-48. https://doi.org/10.1525/bio.2013.63.1.9.

Allagnat, L., Berghmans, S., Falk-Krzesinski, H. J., Hanafi, S., Herbert, R., Huggett, S., \& Tobin, S. (2017). Gender in the Global Research Landscape. 96. https://www.elsevier.com/research-intelligence/ resource-library/gender-report\%0Ahttps://www.elsevier.com/_data/assets/pdf_file/0008/265661/ ElsevierGenderReport_final_for-web.pdf\%0Ahttps://www.elsevier.com/research-intelligence/ research-initiatives/gender.

Bayazit, K. (2020). The Researcherjourney through a genderlens. Elsevier, 1 (1), 1-180. https://www.elsevier. com/connect/gender-report?SIS_ID=307423\&dgcid=RN_AGCM_Program_300004636\&utm_ medium=email\&utm_term=Mendeley_GR2020\&utm_source=AC_99\&utm_campaign=RN_AGCM_ Program_300004636\%0Ahttps://www.elsevier.com/_data/assets/pdf_file/0012/985674/Ge.

Brown, L., \& Osman, H. (2017). The female tourist experience in Egypt as an Islamic destination. Annals of Tourism Research, 63, 12-22. https://doi.org/10.1016/j.annals.2016.12.005.

Goulden, M., Mason, M. A., \& Frasch, K. (2011). Keeping Women in the Science Pipeline. Annals of the American Academy of Political and Social Science, 638(1), 141-162. https://doi. org/10.1177/0002716211416925.

Guimarães, C. R. F. F., \& Silva, J. R. (2016). Pay gap by gender in the tourism industry of Brazil. Tourism Management, 52, 440-450. https://doi.org/10.1016/j.tourman.2015.07.003.

Hair, J. F. J., Anderson, R. E., Tatham, R. L., \& Black, W. (2005). Análise Multivariada de Dados. Bookman.

Martinez, E. D., Botos, J., Dohoney, K. M., Geiman, T. M., Kolla, S. S., Olivera, A., Qiu, Y., Rayasam, G. V., Stavreva, D. A., \& Cohen-Fix, O. (2007). Falling off the academic bandwagon. Women are more likely to quit at the postdoc to principal investigator transition. EMBO Reports, 8(11), 977-981. https:// doi.org/10.1038/sj.embor.7401110.

Minasi, S. M., \& Censon, D. (2020). Mulheres na academia do turismo no Brasil.

Nunkoo, R., Thelwall, M., Ladsawut, J., \& Goolaup, S. (2020). Three decades of tourism scholarship: Gender, collaboration and research methods. Tourism Management, 78(June 2019), 104056. https:// doi.org/10.1016/j.tourman.2019.104056.

Observatório do Turismo de Minas Gerais, O. (2020). Mulheres no Turismo em Minas Gerais.

Ramalho, M., Martins, C., \& Moreau, C. S. (2020). Myrmecology: majority of females only within the colony. Boletim Do Museu Paraense Emílio Goeldi - Ciências Naturais, 15(1), 17-26. https://doi. org/10.46357/bcnaturais.v15i1.241.

Schaap, J. I., \& Shockley, M. (2020). The Glass Ceiling: Is It Being Shattered? E-Journal of Business and Economic Issues, 1-20. 
Silveira, C. E., Medaglia, J., Shizue, M., \& Nakatani, M. (2020). Artigos O mercado de trabalho dos egressos de cursos superiores em turismo : comparações dos dados de 2012 - 2018 Labour Market for Tourism Graduates : comparisons between 2012 - 2018 data El mercado laboral para egresado de programas superiores de Turism. 14(2), 83-94. https://doi.org/http://dx.doi.org/10.7784/rbtur. v14i2.1779 1.

Swain, M. B. (1995). Gender in tourism. In Annals of Tourism Research (Vol. 22, Issue 2, pp. 247-266).

TurisData UNIRIO. (n.d.). Base de Dados Turismo e Mulheres - Turisdata-RJ. Base de Dados Sobre Os Estudos Do Turismo. Retrieved June 16, 2021, from http://www.unirio.br/turisdata-ri-rj-rj-rj/base-dedados-mulheres-e-turismo.

Walker, E., \& Webster, B. (2004). Gender issues in home-based businesses. Women in Management Review, 19(8), 404-412. https://doi.org/10.1108/09649420410570216.

West, J. D., Jacquet, J., King, M. M., Correll, S. J., \& Bergstrom, C. T. (2013). The Role of Gender in Scholarly Authorship. PLOS ONE, 8(7). https://doi.org/10.1371/journal.pone.0066212.

\section{CONTRIBUIÇÃO DOS AUTORES}

Marina de Freitas Coelho: Organização e redação geral do artigo.

Verônica Feder Mayer: Tema do artigo, análises quantitativas e discussões.

Mariana B. de Andrade Matos: Revisão de literatura e discussões teórico-empíricas.

Daniela Fantoni Alvares: Revisão geral do artigo, discussões teórico-empíricas. 